\title{
The efficacy of gastrodin in combination with folate and vitamin B12 on patients with epilepsy after stroke and its effect on HMGB-1, IL-2 and IL-6 serum levels
}

\author{
HAO ZHOU ${ }^{1}$, NUAN WANG ${ }^{1}$, LEI XU ${ }^{2}$, HONGLI HUANG ${ }^{1}$ and CHUNYU YU ${ }^{1}$ \\ Departments of ${ }^{1}$ Neurology and ${ }^{2}$ Pharmacy, The First People's Hospital of Xuzhou, Xuzhou, Jiangsu 221000, P.R. China
}

Received April 11, 2017; Accepted September 4, 2017

DOI: 10.3892/etm.2017.5116

\begin{abstract}
This study evaluated the efficacy of gastrodin in combination with folate (FOL) and vitamin-B12 (V-B12) on patients with epilepsy after stroke (EAS) and its effect on high-mobility group protein B1 (HMGB-1), interleukin-1 (IL-1), and IL- 6 serum levels. The clinical data of 92 EAS patients admitted for treatment between April, 2014 and March, 2016 were collected. These patients were randomly divided into control and observation groups ( $\mathrm{n}=46$ each) using computer software. Patients in the control group were administered only regular antiepileptic drugs, whereas observation group patients also received a combination of gastrodin, FOL and V-B12. After treatment, we compared efficacy, frequency of epileptic seizure, and Montreal cognitive assessment (MoCA) scores. Serum homocysteine (HCY), FOL and V-B12 levels were detected 3 months later. Enzyme-linked immunosorbent assay (ELISA) was used to detect changes in serum HMGB-1, IL-2 and IL-6 levels at one day before treatment and on the 7th, 21st, 30th and 90th days after treatment. Pearson's correlation coefficient was utilized to analyze the correlations of HMGB-1 with IL-2 and IL-6. The total treatment effectiveness rate was $95.65 \%$ in the observation group, which was significantly higher than the control group $(73.91 \%, \mathrm{p}<0.05)$. Epileptic seizure frequency and MoCA scores significantly improved in the observation group $(\mathrm{p}<0.05)$. Serum HCY levels were significantly lower, whereas FOL and V-B12 serum levels were significantly higher, at 3 months post-treatment start in the observation group relative to control group $(\mathrm{p}<0.05)$. After treatment, serum HMGB-1, IL-2 and IL-6 levels progressively decreased over time in both groups, but observation group levels were significantly lower than in control group $(\mathrm{p}<0.05)$.
\end{abstract}

Correspondence to: Dr Nuan Wang, Department of Neurology, The First People's Hospital of Xuzhou, 19 Zhongshan Road North, Quanshan, Xuzhou, Jiangsu 221000, P.R. China

E-mail: wang_nuan1212@163.com

Key words: gastrodin, folate, vitamin B12, epilepsy after stroke, homocysteine, interleukin-2, interleukin-6, high-mobility group protein $\mathrm{B} 1$
Pearson's correlation coefficient analysis showed that HMGB-1 levels were positively correlated with IL-2 and IL-6 levels. A combination of gastrodin, FOL and V-B12 for EAS can significantly improve inflammatory response symptoms, decrease HCY levels, and increase FOL and V-B12 levels in serum while effectively controlling epileptic seizures, thus exhibiting relatively better clinical efficacy. Therefore, this combination treatment is worthy of being promoted in clinical practice.

\section{Introduction}

As a type of common multi-onset disease, stroke is often characterized by high mortality and morbidity rates. Stroke is generally considered as a major factor causing epilepsy in adults (1). Epilepsy, a type of recurrent cerebral dysfunction disease caused by abnormal neuron discharges in the brain, is divided into subtypes according to discharge site (e.g., frontal lobe epilepsy, temporal lobe epilepsy, centrotemporal benign epilepsy and occipital lobe epilepsy) (2). A few epilepsy after stroke (EAS) patients can have their conditions controlled with only short-term administration of antiepileptic drugs. However, the majority of EAS patients require long-term medication to control epileptic seizures. Generally, monotherapy can be effective, but combination medication is required in many circumstances (3). Gastrodin, an active ingredient from the Gastrodia elata Blume, is used extensively in Chinese medicine, and is characterized by pacification, analgesia, and sedative, effects. Study has shown that gastrodin has antagonistic effects against epilepsy and can extend the epilepsy latency time (4). Folate (FOL) and vitamin-B12 (V-B12) are key coenzymes involved in human HCY metabolism, and are beneficial to neural function recovery in EAS patients (5). Highmobility group protein B1 (HMGB-1), interleukin-2 (IL-2), and IL-6 play key roles in the occurrence and development of EAS by participating in EAS pathology. Here, we investigated the efficacy of gastrodin, FOL and V-B12 combination therapy versus conventional antiepileptic drugs for EAS treatment, and observed relatively satisfactory results.

\section{Materials and methods}

Patient information. We randomly selected 92 EAS patients who received treatment between April, 2014 and March, 2016. 
Table I. General patient data.

\begin{tabular}{lcccr}
\hline & Observation group $(\mathrm{n}=46)$ & Control group $(\mathrm{n}=46)$ & $\mathrm{t} / \chi^{2}$ & P-value \\
\hline Gender (male/female) & $25 / 21$ & $22 / 24$ & 0.174 & 0.676 \\
Age (years) & $40-80$ & $40-85$ & & \\
Average age (years) & $58.34 \pm 8.49$ & $59.15 \pm 7.52$ & 0.484 & 0.629 \\
Disease course (year) & $7.35 \pm 2.49$ & $6.96 \pm 2.37$ & 0.769 & 0.443 \\
Degree of education (n, \%) & & & & \\
$\quad$ Junior middle school or below & $9(19.57)$ & $12(26.09)$ & 0.118 & 0.731 \\
Senior middle school or & $21(45.65)$ & $19(41.30)$ & 0.061 & 0.804 \\
technical secondary school & & & & \\
Junior college or above & $16(34.78)$ & $15(32.61)$ & 0.646 & 0.421 \\
Epilepsy subtype (n, \%) & & & & \\
$\quad$ Temporal lobe epilepsy & $5(10.86)$ & $6(13.04)$ & 0.001 & 0.999 \\
Occipital lobe epilepsy & $13(28.26)$ & $10(21.74)$ & 0.232 & 0.630 \\
Frontal lobe epilepsy & $17(36.96)$ & $11(23.91)$ & 0.045 & 0.831 \\
Centrotemporal benign epilepsy & $13(28.26)$ & 0.056 & 0.812 \\
\hline
\end{tabular}

Inclusion criteria were as follows: i) patients diagnosed as epileptic according to the classification methods stipulated by the International League Against Epilepsy (ILAE) and electroencephalogram (EEG) examinations; ii) patients who had never experienced epilepsy prior to stroke, but suffered from epilepsy several times after the onset of stroke; iii) patients who signed written informed consent. Exclusion criteria were as follows: i) patients diagnosed with secondary epilepsy caused by tumor, brain trauma, history of craniocerebral operation or central nervous system infection; ii) patients who were unable to receive the medication on time; iii) patients who were allergic to the drugs used in this study. The patients were randomly divided into control and observation groups by computer software (SPSS Inc., Chicago, IL, USA) (n=46 each). No statistically significant differences were identified between the two groups in terms of general data ( $p>0.05)$ (Table I). The study was approved by the Ethics Committee of the First People's Hospital of Xuzhou.

\section{Methods}

Antiepileptic drug regimens. Patients in both groups received medication in accordance with their epilepsy type. Patients with partial seizures took carbamazepine (Jiangsu Yellow River Pharmaceutical Limited by Share Ltd. (Jiangsu, China); approval no.: Chinese Drug Approval no. H32020638) at an initial dosage of $100 \mathrm{mg}$ twice daily, which was augmented to $300 \mathrm{mg}$ thrice a day within 1-4 weeks. Patients with generalized seizures took sodium valproate orally [Hunan Xiangzhong Pharmaceutical Co., Ltd. (Hunan, China); approval no.: Chinese Drug Approval no. H43020874] at a dose of $300 \mathrm{mg}$ thrice daily. Patients with absence seizures took lamotrigine [Sanjin Group Hunan Sanjin Pharmaceutical Co., Ltd. (Hunan, China); approval no.: Chinese Drug Approval no. H20050596] at an initial dosage of $25 \mathrm{mg}$ once daily, which was augmented to $50 \mathrm{mg}$ twice a day within 1-4 weeks. Patients with myoclonic seizures took clonazepam [Jiangsu Nhwa Saide Pharmaceutical Co., Ltd. (Jiangsu, China); approval no.: Chinese Drug Approval no. H32020591] at an initial dosage of $0.5 \mathrm{mg}$ thrice per day, which was augmented to $2 \mathrm{mg}$ thrice a day within 1-4 weeks. Patients with generalized tonic clonic seizures took phenytoin sodium [CSPC Ouyi Pharmaceutical Co., Ltd. (Shijiazhuang, China); approval no.: Chinese Drug Approval no. H13020977] at an initial dosage of $100 \mathrm{mg}$ twice daily, which was augmented to $150 \mathrm{mg}$ thrice per day within 1-4 weeks.

Combination medication treatment. In the observation group, patients took gastrodin tablets orally [Tonghua Renmin Pharmaceutical Stock Co., Ltd. (Jilin, China); Approval no.: Chinese Drug Approval no. H22025759] in addition to antiepileptic drugs at a dosage of $50 \mathrm{mg}$ thrice a day. FOL tablets were orally administered daily [Jiangsu Yabang Epson Pharmaceutical Co.,Ltd. (Jiangsu, China); Approval no.: Chinese Drug Approval no. H32023288] at a dose of $5 \mathrm{mg} /$ day. V-B12 tablets were administered [Yunpeng Shanxi Pharmaceutical Co., Ltd. (Shanxi, China); Approval no.: Chinese Drug Approval no. H14023321] at a dosage of $25 \mu \mathrm{g}$ thrice per day.

Detection of serum HCY, FOL and V-B12 levels. After 3 months post-treatment, we collected 3-5 ml of venous blood from patients who did not eat or drink anything for $8 \mathrm{~h}$ prior to collection. Samples, after being placed at room temperature for $1 \mathrm{~h}$, were centrifuged at $4^{\circ} \mathrm{C}$ for $15 \mathrm{~min}$ at $2,100 \mathrm{x} \mathrm{g}$, with the supernatant collected and refrigerated at $-20^{\circ} \mathrm{C}$. Detection of serum HCY levels was performed using an HCY kit (Beijing Wantai DRD Co., Ltd., Beijing, China) in strict accordance with manufacturer's instructions via a biochemical analyzer (Beckman Coulter Co., Ltd., Brea, CA, USA). Additionally, serum FOL and V-B12 levels were detected using FOL and V-B12 detection kits respectively (Beckman Coulter Co., Ltd.) via chemiluminescence in accordance with manufacturer's instructions.

Detection of serum HMGB-1, IL-2 and IL-6 levels. At one day prior to treatment and on the 7th, 21st, 30th and 90th days after treatment, we collected 3-5 ml of venous blood of patients who did not eat or drink anything for $8 \mathrm{~h}$ prior to collection. Samples, after being placed at room temperature for $1 \mathrm{~h}$, were centrifuged at $4^{\circ} \mathrm{C}$ for $20 \mathrm{~min}$ at $1,600 \mathrm{x} \mathrm{g}$, with the supernatant at $-80^{\circ} \mathrm{C}$. Enzyme-linked immunosorbent assay (ELISA) was 
Table II. Comparison of efficacy (n, \%).

\begin{tabular}{lcccccc}
\hline Group & $\mathrm{n}$ & Excellent effectiveness & Effective & Improvement & Ineffective & Total effectiveness \\
\hline Observation & 46 & $34(73.91)$ & $7(15.22)$ & $3(6.52)$ & $2(4.35)$ & $44(95.65)$ \\
Control & 46 & $19(41.30)$ & $9(19.57)$ & $6(13.04)$ & $12(26.09)$ & $34(73.91)$ \\
\hline
\end{tabular}

The ranked sum test comparing efficacy between the two groups showed $\mathrm{Z}=3.434$ and $\mathrm{p}=0.001$.

Table III. Comparison of epileptic seizure frequency and MoCA scores.

\begin{tabular}{|c|c|c|c|c|c|c|c|c|}
\hline \multirow[b]{2}{*}{ Group } & \multicolumn{4}{|c|}{ MoCA (points) } & \multicolumn{4}{|c|}{ Epileptic seizure frequency (time) } \\
\hline & Before treatment & After treatment & t-test & P-value & Before treatment & After treatment & t-test & P-value \\
\hline Observation & $25.4 \pm 2.3$ & $14.5 \pm 1.9$ & 24.781 & $<0.0001$ & $26.2 \pm 3.2$ & $11.2 \pm 3.3$ & 22.132 & $<0.0001$ \\
\hline Control & $25.3 \pm 2.4$ & $18.7 \pm 1.8$ & 14.921 & $<0.0001$ & $25.8 \pm 3.3$ & $17.6 \pm 3.4$ & 21.218 & $<0.0001$ \\
\hline t-test & 0.204 & 10.884 & & & 0.590 & 9.161 & & \\
\hline $\mathrm{P}$-value & 0.838 & $<0.0001$ & & & 0.556 & $<0.0001$ & & \\
\hline
\end{tabular}

MoCA, Montreal cognitive assessment.

Table IV. Comparison of patient HCY, FOL and V-B12 levels.

\begin{tabular}{lcccc}
\hline Group & $\mathrm{n}$ & $\begin{array}{c}\mathrm{HCY} \\
(\mu \mathrm{mol} / \mathrm{l})\end{array}$ & $\begin{array}{c}\mathrm{FOL} \\
(\mu \mathrm{mol} / \mathrm{l})\end{array}$ & $\begin{array}{c}\mathrm{V}-\mathrm{B} 12 \\
(\mathrm{pmol} / \mathrm{l})\end{array}$ \\
\hline Observation & 46 & $12.38 \pm 7.27$ & $16.48 \pm 6.37$ & $213.12 \pm 26.43$ \\
Control & 46 & $26.46 \pm 7.16$ & $8.62 \pm 6.35$ & $126.45 \pm 17.52$ \\
t-test & & 9.359 & 5.297 & 18.538 \\
P-value & & $<0.0001$ & $<0.0001$ & $<0.0001$ \\
\hline
\end{tabular}

HCY, homocysteine; FOL, folate; V-B12, vitamin-B12.

used to detect serum HMGB-1, IL-2 and IL-6 levels, and all kits used in this procedure were manufactured by Sangon Biotech Shanghai Co., Ltd. (Shanghai, China). All operations were in strict accordance with manufacturer's instructions. OD values at a wavelength of $450 \mathrm{~nm}$ were detected using a microplate reader (Jiangsu Potebio Co., Ltd., Jiangsu, China) and concentrations of HMGB-1, IL-2 and IL-6 were calculated accordingly.

Observation and follow-up. After medication, patient adverse reactions, such as gastrointestinal reactions, dizziness, changes in weight, dermatitis, somnolence and mental symptoms, were observed at all times. Mild symptoms were alleviated via dosage adjustments, while patients with severe symptoms were delivered to the hospital for treatment. A 1-year follow-up was carried out for all patients discharged from the hospital. Long-term medication was required for patients, and dosage reduction and/or drug withdrawal was carried out in accordance with the principal of gradual reduction only after epilepsy was fully controlled.

\section{Evaluation indexes}

Evaluation criteria for efficacy of treatment: i) Excellent effectiveness: Seizure frequency was reduced by $75-99 \%$ within
6 months. ii) Effective: Seizure frequency was reduced by 50-74\% within 6 months. iii) Improvement: Seizure frequency was reduced by $25-49 \%$ within 6 months. iv) Ineffective: Seizure frequency was reduced by $<25 \%$ or increased within 6 months. Total effectiveness rate $=$ ('excellent effectiveness' rate + 'effective' rate + 'improvement' rate) $\times 100 \%$.

Comparisons were performed regarding patient epileptic seizure frequencies and Montreal cognitive assessment (MoCA) scores. Twenty-eight days was set as the unit of seizure frequency. The MoCA scale was used to evaluate patient condition in 8 domains (spatial and executive abilities, memory, attention and concentration, naming, language, recall, abstract thinking and orientation) before and after treatment, with a maximum score of 30 points.

Statistical analysis. Data were processed using SPSS 19.0 software (SPSS Inc., Chicago, IL, USA). Measurement data were presented as mean \pm standard deviation (SD), and t-tests were performed. Ranked sum tests were adopted in comparisons of treatment efficacy, and Pearson's correlation coefficient was adopted in correlation analysis. $\mathrm{p}<0.05$ is considered to indicate a statistically significant difference.

\section{Results}

After 6 months of treatment, we compared treatment efficacy between the two groups, and found that total treatment effectiveness rate was $95.65 \%$ in the observation group, which was significantly higher than the control group $(73.91 \%$, $\mathrm{p}<0.05$ ) (Table II).

Comparison of epileptic seizure frequency and MoCA scores: At 6 months post-treatment, ameliorations were detected in patient epileptic seizure frequency and MoCA scores in both groups. The observation group showed significantly better improvement in both parameters compared to the control group $(\mathrm{p}<0.05)$ (Table III). 


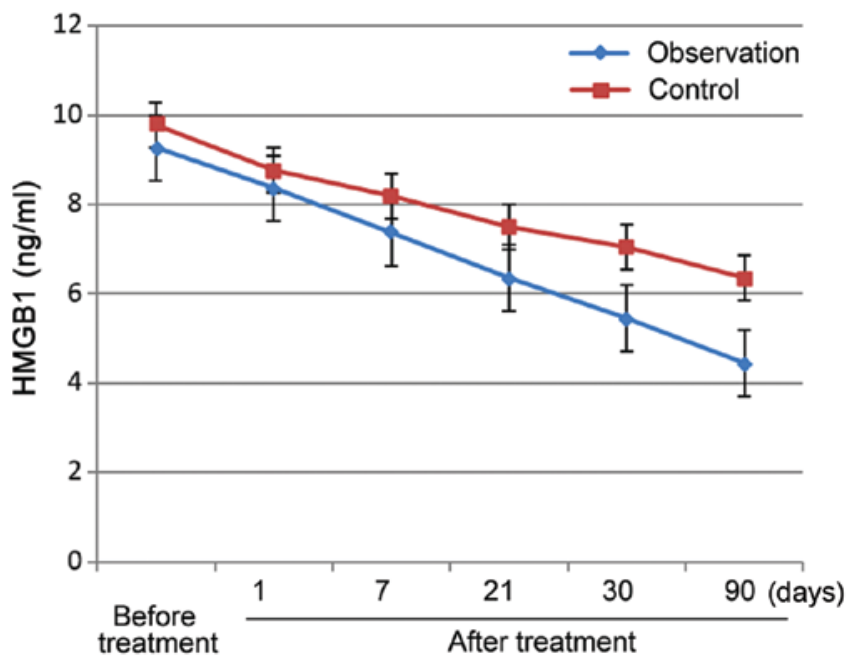

Figure 1. Change in patient serum high-mobility group protein B1 (HMGB-1) levels.

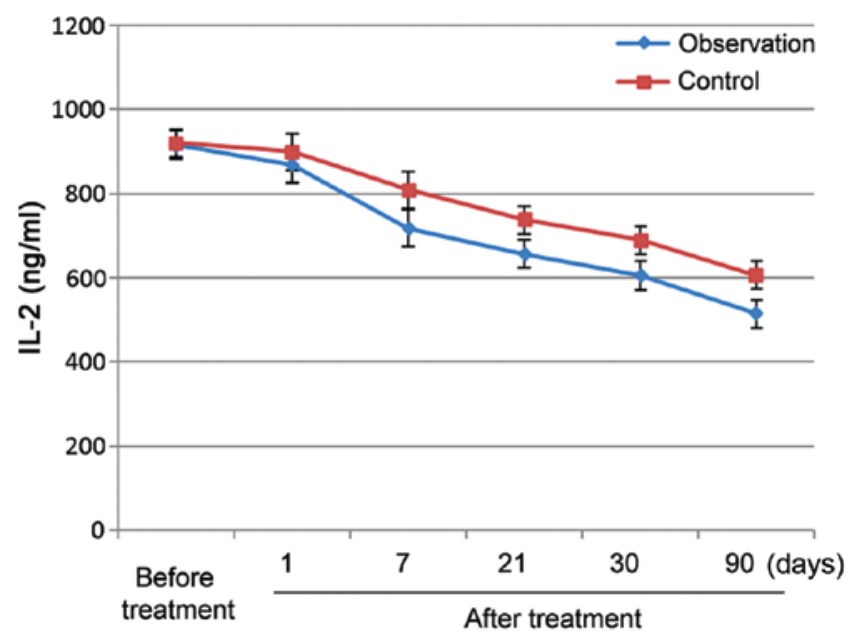

Figure 2. Change in patient serum interleukin-2 (IL-2) levels.

At 3 months after treatment, analysis of serum HCY, FOL and V-B12 levels showed that serum HCY levels in observation group patients were significantly lower than control group patients. Serum FOL and V-B12 levels in the observation group were significantly higher than in the control group $(\mathrm{p}<0.05)$ (Table IV).

After treatment, serum HMGB-1, IL-2 and IL-6 levels decreased in both groups, and this decreasing trend persisted over time. Observation group levels were significantly lower for all three parameters than control group levels $(\mathrm{p}<0.05)$ (Figs. 1-3).

Correlation analysis for HMGB-1 levels and IL-2 and IL-6 levels. Pearson's correlation coefficient analysis revealed that HMGB-1 levels were positively correlated with IL-2 and IL-6 levels $(\mathrm{p}<0.05)$ (Table V and Fig. 4).

\section{Discussion}

Features and influencing factors of EAS. Stroke is one of the major factors leading to adult epilepsy. Epilepsy can be divided into two categories based on onset time: early-onset epilepsy

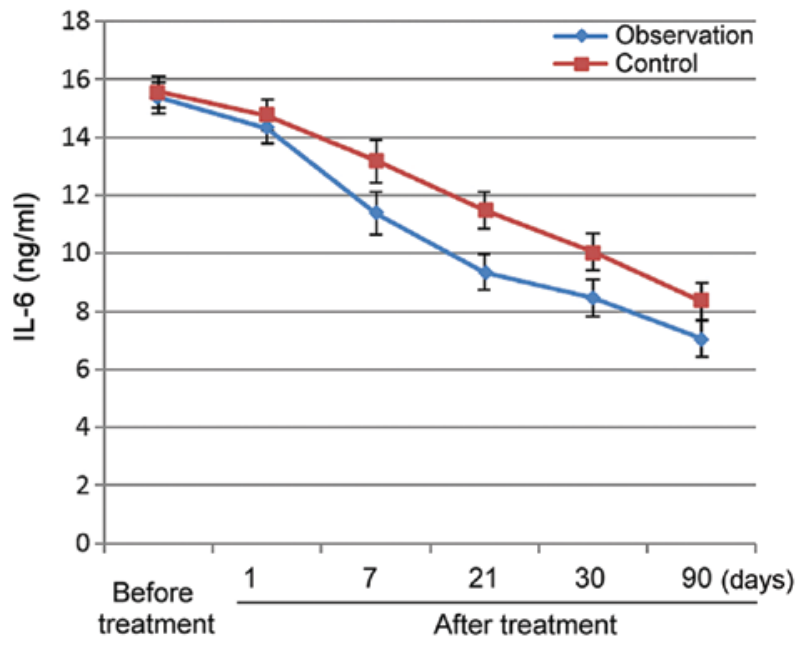

Figure 3. Change in patient serum interleukin-6 (IL-6) levels.

Table V. Correlation analysis for HMGB-1 levels and IL-2 and IL-6 levels.

\begin{tabular}{lcc}
\hline Item & Relevant coefficient & P-value \\
\hline IL-2 & 0.774 & 0.005 \\
IL-6 & 0.627 & 0.013 \\
\hline
\end{tabular}

HMGB-1, high-mobility group protein B1; IL, interleukin.

after stroke and late-onset epilepsy after stroke, with the cut-off point at usually around 2 weeks (6). EAS can greatly affect patient neurological function, which deleteriously impacts regular recovery and can even lead to deterioration or death. Post-stroke injury to the brain structure and function may induce neuronal changes, necrosis, and/or deficiency, causing neurotransmitter balance alterations, increased excitability, abnormal ion distribution, and excessive cerebral neuron discharge, thus resulting in deterioration of epilepsy or even persistent epilepsy, which threatens the lives of patients and forms a vicious circle (7). Additionally, EAS is characterized by transient, abrupt, and recurrent features. The onset of EAS may be associated with following factors: i) glial tissue hyperplasia: Which can induce apoplectic cyst formation, which replaces normal brain tissue to decrease neural cell regulatory functions, weakens excitability regulation, and causes excessive neuron discharge, thus resulting in epilepsy (8). ii) Factors affecting neural cell membrane stability: after stroke, neural cell membrane instability can give rise to abnormalities in the function of surrounding cells and selective neuronal mutations, leading to an increase in neural cell excitability and synchronous discharge, thus resulting in epilepsy (9). iii) Inflammatory factors: after stroke, long-term inflammatory responses can generate changes in morphology, increasing the amount of neuroglia, thus causing or aggravating epilepsy (10). iv) Immunological factors: Disorders in immunological functions have been found in EAS patients, including abnormal humoral and cellular immunological functions, which can induce the onset of epilepsy (11). 

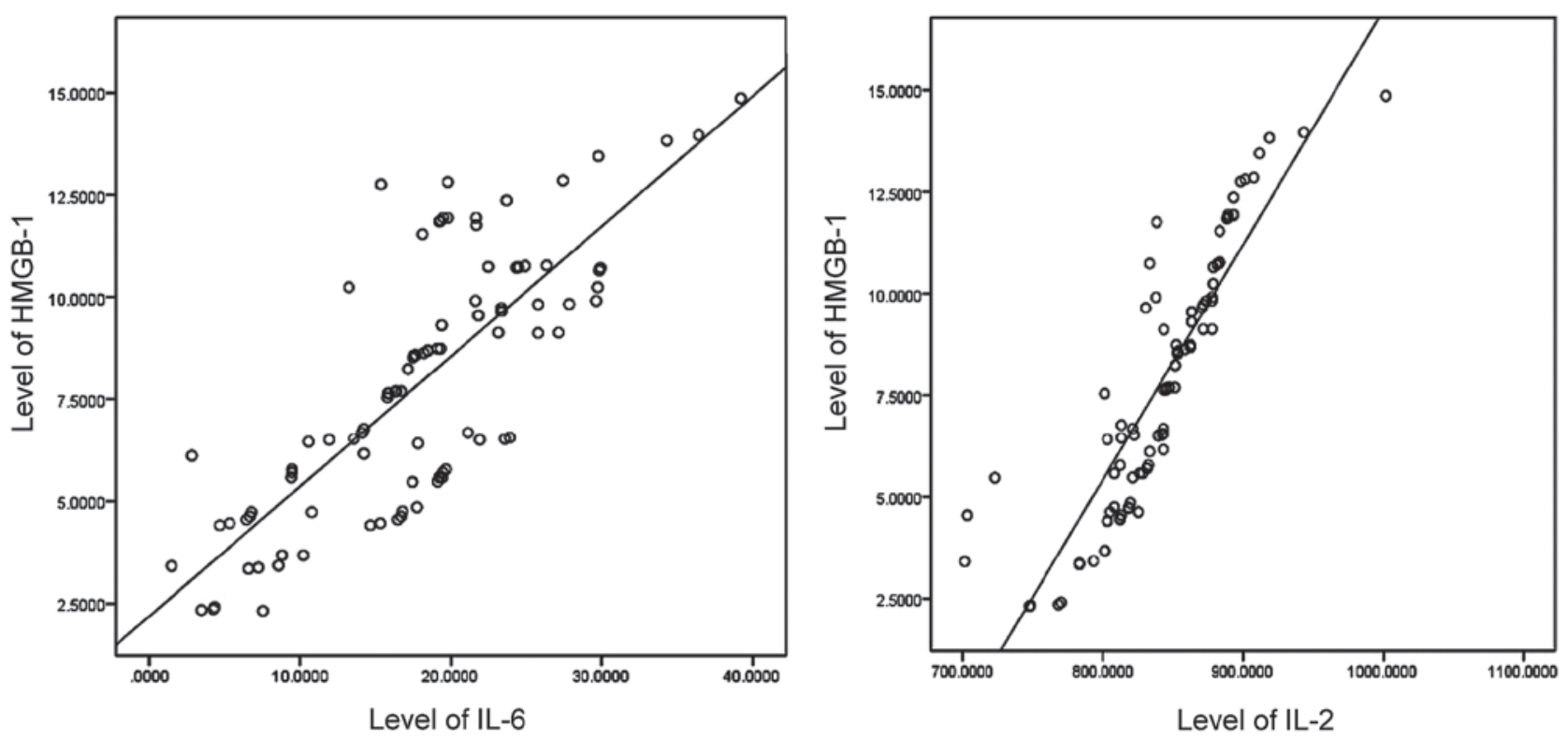

Figure 4. Scatter diagram of the correlation between high-mobility group protein B1 (HMGB-1) and interleukin-6 (IL-6) levels and between HMGB-1 and IL-2 levels. Pearson's correlation coefficient analysis showed $r=0.774$ and $\mathrm{p}=0.005$, indicating a positive correlation between HMGB-1 levels and IL-2 and IL-6 levels.

EAS treatment. So far, the pathogenesis of EAS remains unclear, and treatment has also presented problems. Generally, EAS treatment is focused on the primary disease and customized medication can be additionally used based on the features of the epileptic seizures present. Long-term medication is also required, mainly carbamazepine and sodium valproate (12). EAS patients are susceptible to shortages of FOL and V-B12, which can cause disorders in HCY metabolism, leading to increased HCY levels. Thus, medication should be used to combat this condition in specific treatments (13). This study showed that supplementation of FOL and V-B12 significantly reduced serum HCY levels, suggesting that this medication schedule, from the perspective of improvement in primary disease, is beneficial to the treatment and control of epilepsy.

Gastrodin mechanism of action in EAS. Gastrodin is a major active ingredient in Gastrodia elata Blume. Study has indicated that gastrodin, with anticonvulsive and antiepileptic functions, can prolong the latency of epileptic seizures, shorten onset course, alleviate degree of attack, accelerate recovery and decrease mortality rate. Thus, gastrodin can be used as an auxiliary drug to antagonize absence seizures and severe grand mal seizures for the amelioration of patient clinical symptoms (14). The potential mechanism of action of gastrodin may lie in an inhibition of the action and expression of inhibitory and amino acid neurotransmitters (glutamic acid) in the temporal lobe and hippocampus. This suppresses abnormal gap junction formation and decreases cerebral cortex excitability, thus generating the antiepileptic effect. Gastrodin can also alleviate damage to endothelial cells, astrocytes, and neurons in hippocampal vasculature, which can protect brain tissue (15). After combination therapy using gastrodin and antiepileptic drugs, the total treatment effectiveness rate significantly increased $(95.74 \%$ in the observation group vs. $74.47 \%$ in the control group, $\mathrm{p}<0.05$ ).
Significance of serum HMGB-1, IL-2, and IL-6 after EAS. HMGB-1, as a type of nonhistone chromosomal binding protein, is involved in the pathogenesis of various diseases, including tissue damage, respiratory system diseases, sepsis, ischemic brain damage and carcinoma (16). Study has indicated that HMGB-1 expression is elevated inside and outside astrocytes and hippocampal neurons in epilepsy patients (17). IL-6 is an early-stage inflammatory factor, and despite its protective effect on brain tissue, long-term overexposure will not only result in the augmentation of neuroglia, but also cause variations in tissue morphology, thus aggravating epilepsy (18). IL-2 can regulate the balance of lymphocytes, shorten epilepsy latency and decrease the threshold value for extending the onset of epileptic discharge (19). This study showed that serum HMGB-1 levels were positively correlated with the levels of IL-2 and IL-6. HMGB-1 functions as a central link in the network of pro-inflammatory factors, the release of HMGB-1 can induce the secretion of IL-2 and IL-6 for the amplification of inflammatory responses, while IL-2 and IL-6 can in turn promote the release of HMGB-1, thus forming positive feedback loops (20). After treatment, patient serum levels of HMGB-1, IL-2 and IL-6 in the observation group were significantly lower than in the control group, suggesting that this medication schedule was beneficial to the control of epilepsy.

In conclusion, treatment with a combination of gastrodin, FOL, and V-B12 in addition to standard antiepileptic treatment can effectively control epileptic seizures and increase treatment efficacy for EAS patients.

\section{Acknowledgements}

This study was supported by the Clinical Science and Technology Development Fund of Jiangsu University (no. JLY20160140) and the Xuzhou Science and Technology Planning Project (no. KC16SH025). 


\section{References}

1. Keller L, Hobohm C, Zeynalova S, Classen J and Baum P: Does treatment with t-PA increase the risk of developing epilepsy after stroke? J Neurol 262: 2364-2372, 2015.

2. Sharma R and Pachori RB: Classification of epileptic seizures in EEG signals based on phase space representation of intrinsic mode functions. Expert Syst Appl 42: 1106-1117, 2014.

3. Schmidt D and Schachter SC: Drug treatment of epilepsy in adults. BMJ 348: g254-g254, 2014.

4. Zhang R, Peng Z, Wang H, Xue F, Chen Y, Wang Y, Wang H and Tan Q: Gastrodin ameliorates depressive-like behaviors and up-regulates the expression of BDNF in the hippocampus and hippocampal-derived astrocyte of rats. Neurochem Res 39: 172-179, 2014.

5. Eldayem SMA, Saleh ON, Emara NA, Kandil ME and Shatla RH: Evaluation of homocysteine, folate and vitamin B12 levels among Egyptian children with idiopathic epilepsy. Maced J Med Sci 7: 109-113, 2014.

6. Hsu CJ, Weng WC, Peng SS and Lee WT: Early-onset seizures are correlated with late-onset seizures in children with arterial ischemic stroke. Stroke 45: 1161-1163, 2014.

7. Beghi E, D'Alessandro R, Beretta S, Consoli D, Crespi V, Delaj L, Gandolfo C, Greco G, La Neve A, Manfredi M, et al; Epistroke Group: Incidence and predictors of acute symptomatic seizures after stroke. Neurology 77: 1785-1793, 2011.

8. Hattiangady B and Shetty AK: Neural stem cell grafting in an animal model of chronic temporal lobe epilepsy. Curr Protoc Stem Cell Biol: Chapter 2: Unit2D.7, 2011.

9. Bouthour W, Leroy F, Emmanuelli C, Carnaud M, Dahan M Poncer JC and Lévi S: A human mutation in Gabrg2 associated with generalized epilepsy alters the membrane dynamics of GABAA receptors. Cereb Cortex 22: 1542-1553, 2012.

10. Yu N, Liu H and Di Q: Modulation of immunity and the inflammatory response: A new target for treating drug-resistant epilepsy. Curr Neuropharmacol 11: 114-127, 2013.

11. Kan AA, van Erp S, Derijck AAHA, de Wit M, Hessel EV, O'Duibhir E, de Jager W, Van Rijen PC, Gosselaar PH, de Graan PN, et al: Genome-wide microRNA profiling of human temporal lobe epilepsy identifies modulators of the immune response. Cell Mol Life Sci 69: 3127-3145, 2012.
12. Kwan J, Wood E, Kinton L and Cordonnier C: Antiepileptic drugs for the primary and secondary prevention of seizures after stroke. Cochrane Database Syst Rev 1: CD005398-CD005398, 2010.

13. Wang YP, Lin HP, Chen HM, Kuo YS, Lang MJ and Sun A: Hemoglobin, iron, and vitamin B12 deficiencies and high blood homocysteine levels in patients with anti-thyroid autoantibodies. J Formos Med Assoc 113: 155-160, 2014.

14. Tang C, Wang L, Liu X, Cheng M, Qu Y and Xiao H: Comparative pharmacokinetics of gastrodin in rats after intragastric administration of free gastrodin, parishin and Gastrodia elata extract. J Ethnopharmacol 176: 49-54, 2015.

15. Hsieh CL, Chen CL, Tang NY, Chuang CM, Hsieh CT, Chiang SY, Lin JG and Hsu SF: Gastrodia elata BL mediates the suppression of nNOS and microglia activation to protect against neuronal damage in kainic acid-treated rats. Am J Chin Med 33: 599-611, 2005.

16. Li Y, Tian J, Fu X, Chen Y, Zhang W, Yao H and Hao Q: Serum high mobility group box protein 1 as a clinical marker for ovarian cancer. Neoplasma 61: 579-584, 2014.

17. Huang JS, Wu Y, Huang Q, Li SJ, Ye JM, Wei X, Liu QD, Liu Y and Ma MG: Expression level and distribution of HMGB-1 in Sombati's cell model and kainic acid-induced epilepsy model. Eur Rev Med Pharmacol Sci 19: 2928-2933, 2015.

18. Uludag IF, Duksal T, Tiftikcioglu BI, Zorlu Y, Ozkaya F and Kirkali G: IL-1 $\beta$, IL-6 and IL1Ra levels in temporal lobe epilepsy. Seizure 26: 22-25, 2015.

19. Steinborn B, Zarowski M, Winczewska-Wiktor A, Wójcicka M, Młodzikowska-Albrecht J and Losy J: Concentration of IL-1 $\beta$, IL-2, IL-6, TNF $\alpha$ in the blood serum in children with generalized epilepsy treated by valproate. Pharmacol Rep 66: 972-975, 2014.

20. Kwak MS, Lim M, Lee YJ, Lee HS, Kim YH, Youn JH, Choi JE and Shin JS: HMGB-1 binds to lipoteichoic acid and enhances TNF- $\alpha$ and IL- 6 production through HMGB-1-mediated Transfer of lipoteichoic acid to CD14 and TLR2. J Innate Immun 7: 405-416, 2015. 